\title{
Detección de la alteración del metabolismo glucídico y resistencia a la insulina en una muestra piloto infantil: Aproximación metabolómica
}

\author{
Detection of impaired glucidic metabolism and insulin resistance in a pilot child sample \\ Ismael San Mauro-Martin ${ }^{*}$ orcid.org/0000-0002-7415-0293 \\ Sara López-Oliva' ${ }^{1}$ orcid.org/0000-0003-2639-6800 \\ Elena Garicano-Vilar1 orcid.org/0000-0001-6327-6758 \\ Belén García-de Angulo' orcid.org/0000-0003-2257-0621 \\ Javier Andrés Blumenfeld-Olivares² orcid.org/0000-0002-7168-840X
}

1 Centros de Investigación en Nutrición y Salud. Madrid, España

2 Servicio de Endocrinología Pediátrica, Hospital El Escorial. Madrid, España

San Mauro-Martin I, López-Oliva S, Garicano-Vilar E, García-de Angulo B, Blumenfeld-Olivares JA. Detección de la alteración del metabolismo glucídico y resistencia a la insulina en una muestra piloto infantil: Aproximación metabolómica. Univ. Salud. 2019;21(3):191197. DOI: http://dx.doi.org/10.22267/rus.192103.155

\section{Resumen}

Introducción: La metabolómica permite estudiar la resistencia a insulina (RI), un factor de riesgo de pre-diabetes y diabetes. Quantose $I R^{T M}$ es el único test que mide la RI mediante la abrazadera hiperinsulinémica euglicémica. Objetivo: Se comprobó la eficacia de un test metabolómico en la detección de marcadores de RI en población infantil. Materiales y métodos: Once niños, de edad 8,54 $\pm 3,53$ años y con factores de riesgo de diabetes, fueron reclutados del Hospital El Escorial. Se estableció como criterio diagnóstico para la prediabetes el estándar de la Asociación Americana de Diabetes (ADA) (HbA1C 5,7-6,4\% y glucosa basal 100$125 \mathrm{mg} / \mathrm{dl}$ ). Se compararon las analíticas de sangre con la prueba de Quantose $I R^{T M}$, estudiando el perfil del metaboloma relacionado con la RI (ácido alfa-hidroxibutírico, ácido oleico, linoleo-glicerofosfocolina e insulina). Su análisis generó una puntuación Quantose ${ }^{\complement}$ (escala 0-100), siendo >63 RI. Resultados: Ningún sujeto cumplió el criterio de la ADA para prediabetes: HbA1C fue 5,3 $\pm 0,18 \%$ y glucosa $86,6 \pm 5,6 \mathrm{mg} / \mathrm{dl}$. Por el contrario, 10 sujetos cumplieron criterios del test Quantose $I R^{T M}$ para la RI (score: 78,09 $\pm 9,24$ (>63)). Conclusiones: El test Quantose $I R^{T M}$ mide el porcentaje de hemoglobina unida a glucosa dentro de los glóbulos rojos. Permite prever el riesgo de diabetes, y tomar medidas preventivas.

Palabras clave: Diabetes mellitus; resistencia a la insulina; metabolómica; metabolismo de los hidratos de carbono; niño. (Fuente: DeCS, Bireme).

\begin{abstract}
Introduction: Metabolomics enables the study of insulin resistance (IR), a risk factor for pre-diabetes and diabetes. Quantose IRTM is the only test that measures IR using the euglycemic hyperinsulinemic clamp. Objective: The effectiveness of a metabolomic test for the detection of RI markers in a child population was verified. Materials and methods: Eleven children aged $8.54 \pm 3.53$ years with diabetes risk factors were recruited from the El Escorial Hospital. The American Diabetes Association (ADA) Standards (5,76,4\% HbA1C and 100-125 mg/dl basal glucose) were established as diagnostic criteria for prediabetes. Blood tests were compared to the Quantose IRTM assay studying the metabolomic profile related to IR (alpha-hydroxybutyric acid, oleic acid, linoleoglycerophosphocoline and insulin). This analysis generated a Quantose ${ }^{\circledR}$ score of IR $>63$. Results: None of the subjects met the ADA criteria for prediabetes: $\mathrm{HbA} 1 \mathrm{C}=5,3 \pm 0,18$ and glucose $=86,6 \pm 5,6 \mathrm{mg} / \mathrm{dl}$. On the contrary, 10 subjects met the Quantose IRTM test criterion for IR (score: 78,09 $\pm 9,24(>63)$ ). Conclusions: The Quantose IRTM test measures the percentage of glucose bound hemoglobin within red blood cells. This assay makes it possible to predict diabetes risk and take preventive measures.
\end{abstract}

Key words: Diabetes mellitus; insulin resistance; metabolomics; carbohydrate metabolism; child. (Source: DeCS, Bireme). 


\section{Introducción}

El síndrome de resistencia a la insulina (RI) es actualmente uno de los factores etiológicos más importantes tanto de morbilidad, como de mortalidad a nivel mundial, debido a su asociación con obesidad, hipertensión arterial, dislipidemia, arteriosclerosis y el desarrollo de diabetes mellitus (DM) tipo 2(1). Las tasas de prevalencia global de RI, en estudios basados en población de niños y adolescentes, oscilan entre el 3,1 y el $44 \%(2)$.

La RI se define como una reducción en la respuesta fisiológica de los tejidos a la acción de la insulina, particularmente a nivel del tejido muscular y adiposo. La insulina es una hormona anabólica secretada por las células $\beta$ del páncreas en respuesta a diversos estímulos, siendo la glucosa el estímulo más relevante ${ }^{(3)}$. Su principal función es mantener la homeostasis glicémica y de otros sustratos energéticos. De esta forma, posterior a cada comida la insulina suprime la liberación de ácidos grasos libres mientras que favorece la síntesis de triglicéridos en el tejido adiposo. Por otra parte, inhibe la producción hepática de glucosa, mientras que promueve la captación de glucosa por el tejido muscular esquelético y adiposo(4). En un estado de RI, la acción de esta hormona a nivel celular está reducida, aumentando la secreción de insulina. Esto permite compensar el defecto en la acción tisular y así mantener la homeostasis glicémica(5). Este fenómeno da cuenta del estado hiperinsulinémico, el cual es característico en sujetos con RI. Se estima que aproximadamente el $55 \%$ de la variabilidad de la sensibilidad a la insulina en los niños está determinada por el contenido de tejido adiposo y el sexo(6). La edad y el estadio puberal tienen un importante impacto en la distribución de la grasa y en la sensibilidad a la acción de la insulina(7).

El incremento del depósito de grasa visceral es el principal factor independiente de riesgo en el desarrollo de estados de RI en niños y adolescentes ${ }^{(8)}$. La nutrición puede ser un factor favorecedor del desarrollo de RI. Así se ve favorecida por las dietas hipercalóricas con un elevado contenido de grasa y carbohidratos y con un bajo aporte de fibra ${ }^{(9)}$.

Hay un creciente reconocimiento de RI en la patogénesis de la diabetes tipo 2 en niños(10). En poblaciones pediátricas obesas la existencia de RI puede ser el punto de partida para el desarrollo posterior de diabetes tipo 2 y/o síndrome metabólico(11), habiéndose definido recientemente la prediabetes tipo 2 en el adolescente obeso(12).

El aumento en la prevalencia de obesidad se ha visto acompañado de un incremento en la incidencia de diabetes tipo 2 en la población pediátrica(13). El aumento del IMC en niños se asocia con mayor RI(14). Las complicaciones metabólicas y cardiovasculares de la obesidad están estrechamente relacionadas con la presencia de hiperinsulinemia y de RI(15). Dado el alarmante aumento de las tasas de obesidad entre los jóvenes, existe una gran preocupación de que la incidencia de diabetes sombreará esta tendencia. Lo que hace que la pandemia sea particularmente amenazante es que, en gran parte del mundo, permanece oculta ( 1 de cada 2 diabéticos están sin diagnosticar)(16). Durante décadas, la glucosa, la hemoglobina A1c, la insulina y el péptido $C$ han sido las pruebas de laboratorio de elección para detectar y vigilar la diabetes. Sin embargo, estas pruebas no identifican a los individuos prediabéticos o sus subfenotipos que están en riesgo de desarrollar DM tipo 2, este sería un requisito para la prevención individualizada(17).

Los métodos diagnósticos disponibles para medir la sensibilidad a la insulina en la población pediátrica son: 1) el clamp hiperinsulinémicoeuglucémico, que representa el estándar de oro para medir la sensibilidad tisular a la insulina y la secreción de insulina aunque es de elevada complejidad(18); 2) los índices de HOMA (Homeostasis Model Assessment)(19) y QUICKI (Quantitative Insulin Check Index)(20), que son los métodos más sencillos y los más utilizado para evaluar RI en el campo; y 3) el índice MatsudaDeFronzo (también llamado ISI-Compuesto) que se calcula a partir de una curva de tolerancia a la glucosa oral (CTGO) de la cual se desprende información adicional sobre el metabolismo de la glucosa en el estado post-estimulatorio(21).

La prueba de tolerancia oral a la glucosa sigue siendo el único medio para la identificación precoz y fiable de personas en la fase de prediabetes con alteración de la tolerancia a la glucosa. Este procedimiento, sin embargo, es muy largo $y$ costoso $y$ no es recomendable como método de cribado en una consulta médica. Por lo tanto, existe la necesidad de pruebas de laboratorio innovadoras para simplificar la detección precoz de las alteraciones en el metabolismo de la glucosa(17). Las nuevas tecnologías 
para el diagnóstico, como la metabolómica, son herramientas sensibles, específicas y de gran utilidad frente a otras técnicas modernas, como la genómica.

El test Quantose ${ }^{T M} I R$ (Metabolom INC, USA) es una prueba basada en la metabolómica para analizar la RI, capaz de generar un valor como indicador precoz de riesgo para el desarrollo de la prediabetes y la DM tipo 2(22). El $\alpha$-hidroxibutirato es el metabolito más importante asociado con la sensibilidad a la insulina y es considerado un marcador precoz tanto para la RI como para la disglucemia, alteración de la regulación de la glucosa(23).

Quantose ${ }^{T M} I R$ es un análisis de sangre en ayunas que mide un panel de biomarcadores, que son reflejo de la RI. El panel de biomarcadores comprende un ácido orgánico (ácido alfa-hidroxibutírico), dos lípidos (ácido oleico y linoleoilglicerofosfocolina) y la insulina. La puntuación en la prueba fue desarrollada para estimar el valor obtenido del clamp euglucémico hiperinsulinémico, el estándar de oro para determinar la sensibilidad a la insulina, en un estudio prospectivo, observacional de 1277 personas clínicamente sanas, no diabéticas reclutadas en 13 países europeos(22). El punto de corte en 63 fue definido por el tercil superior de puntuaciones en el estudio. Los niveles de insulina son medidos por inmunoquimioluminiscencia, y los 3 metabolitos son detectados por espectrometría de masas/cromatografía liquída (UHPLC LC-MS / MS). Las concentraciones de los 4 biomarcadores se combinan y analizan en un algoritmo que genera la puntuación de Quantose IR (escala 1-120).

La mayoría de los casos de DM tipo 2 pueden ser detectados y tratados oportunamente e invertir en nuevas tecnologías como método de prevención y detección precoz, puede reducir la carga mundial de diabetes y ahorrará miles de millones en pérdida de productividad y costes sanitarios(16). Por lo anterior, este estudio tiene por objetivo comprobar la efectividad de un test metabolómico en la detección de marcadores de RI en población infantil.

\section{Materiales y métodos}

Estudio piloto descriptivo, transversal, ambispectivo, cuya población estuvo constituida por 11 niños (8 hombres, 3 mujeres) del servicio de pediatría del Hospital San Lorenzo del Escorial (Madrid) con factores de riesgo de complicaciones metabólicas (antecedentes familiares, obesidad, alteración e incremento de glucosa basal en plasma). La muestra fue incidental, pretendiendo tener una primera aproximación del uso (efectividad clínico-económico) de un test comercial, para la posible implementación futura en la práctica clínica.

\section{Parámetros bioquímicos}

Se extrajo sangre venosa en tubos EDTA vacutainer tras un periodo de 12 horas de ayuno de los sujetos. De esta forma se determinó la glucosa mediante el uso de un método de glucosa oxidasa automatizado (Roche Hitachi ${ }^{\circledR}$ 917, Roche Diagnostics, Mannheim, Alemania); insulina por radioinmunoensayo (RIA); hemoglobina glicada (HbA1c) por cromatografía liquida de alta precisión.

Se estableció como criterio diagnóstico para la prediabetes el estándar de la Asociación Americana de Diabetes(24): la HbA1C de 5,7-6,4\% y la glucosa basal $100-125 \mathrm{mg} / \mathrm{dl}$, es decir, superar o no superar esas cifras en alguno de los metabolitos clásicos.

Los criterios de inclusión fueron: Niños de 6 a 12 años, de ambos sexos, sin maduración sexual, con y sin obesidad o sobrepeso, con al menos dos factores de riesgo asociados a la RI (glucemias elevadas en sangre, herencia familiar, sobrepeso u obesidad).

Los criterios de exclusión fueron: Niños con maduración sexual en estadio de Tanner II o superior. Sujetos con diabetes tipo I, pancreatitis, hepatitis, parálisis cerebral, cáncer, enfermedades neuromusculares y psicológicas, u otras enfermedades graves. Sujetos con tratamiento farmacológico o suplemento dietético para el control de la glucemia.

Todos los participantes fueron invitados a participar durante un periodo de 3 semanas e informados de forma escrita y oral, sobre la finalidad del estudio. Los padres de aquellos que decidieron involucrarse firmaron una hoja de consentimiento informado, la cual detallaba los objetivos de la investigación, todos los procedimientos efectuados, la duración prevista de la misma, la falta de certeza sobre la seguridad y eficacia del tratamiento y los datos de los responsables de la investigación.

Del reclutamiento inicial de 15 sujetos, se excluyeron 4 participantes, 2 por no completar las pruebas, 1 por voluntad de los padres, y 1 por fallo en la 
determinación bioquímica en el laboratorio. $\mathrm{Al}$ tratarse de una muestra incidental desde la consulta hospitalaria y voluntaria, no se obtuvo un número equitativo por sexo ni edades.

Los datos fueron recogidos por dietistasnutricionistas entrenados y formados, homogeneizando un protocolo de recolección de datos y monitorización del estudio. Se midió el peso (en kg), la talla (en m), el índice de masa corporal (IMC), el porcentaje de grasa corporal total, el porcentaje de grasa visceral, la masa libre de grasa (en $\mathrm{kg}$ ) y el perímetro de cintura (en $\mathrm{cm}$ ) de cada participante.

El peso, el IMC y la composición corporal se determinaron a través de una bioimepadancia eléctrica modelo Tanita BP601, tetrapolar, multifrecuencia, y una cinta métrica flexible, no elástica, metálica y de anchura inferior a $0,1 \mathrm{~mm}$ $150 \mathrm{~cm}$. Para la realización de la bioimepadancia se siguió el protocolo estándar para este equipo(25) y las recomendaciones del fabricante(26). Dicho protocolo, fue comunicado previamente a los usuarios, para garantizar mayor fiabilidad del resultado.

Además, se realizó un cuestionario ad hoc que se diseñó con base en distintos cuestionarios que incluían datos personales, anamnesis clínica, hábitos de vida, ejercicio y alimentación, con el Cuestionario de Frecuencia de Consumo de Alimentos, suponiendo un tiempo aproximado de resolución entre 5 y 10 minutos.

Posteriormente, se realizó el test Quantose $I R^{T M}$, mediante extracción de sangre, con el que se midió la concentración de cuatro metabolitos relacionados con la RI: alfa-hidroxibutirato, linoleo- glicerofosfocolina, ácido oleico e insulina, detectados por espectrometría de masas/cromatografía líquida (UHPLC LC-MS / MS). Estas mediciones cuantitativas absolutas se utilizan para generar una puntuación Quantose $^{\mathrm{TM}}$ (escala 0-100), donde los valores superiores a 63 son indicativos de RI, y por tanto, prediabetes. La puntuación Quantose ${ }^{\mathrm{TM}}$ también se proporciona en una escala codificada con color. Cuando las puntuaciones Quantose ${ }^{\mathrm{TM}}$ cambian de valores menor a mayores, el color cambia de verde a naranja-rojo, lo que indica el cambio de un paciente sensible a la insulina a resistente a la insulina(27).

El análisis de los datos se llevó a cabo con el programa de análisis estadístico SPSS 20, para los resultados de las mediciones cuantitativas de los 4 metabolitos se combinaron en un algoritmo de regresión logística para generar una puntuación Quantose ${ }^{\mathrm{TM}}$, que supera a otras estimaciones comúnmente utilizadas para la RI.

Consideraciones éticas: El estudio fue aprobado por el Comité de bioética del Hospital Puerta de Hierro de Madrid. Se siguieron las normas éticas del comité encargado de supervisar los ensayos en personas y la declaración de Helsinki de 1975 modificada en 2004.

\section{Resultados}

La muestra estuvo constituida por 11 niños (8 hombres y 3 mujeres) con alto riesgo de diabetes. La edad media fue de 8,54 $\pm 3,53$ años.

De los 11 sujetos, ninguno superó el criterio de la Asociación Americana de Diabetes para prediabetes (5,7-6,4 \% HbA1C y 100-125 mg/dl glucosa basal): la media de la HbA1C fue 5,3 $\pm 0,19 \%$ y de la glucosa $86,6 \pm 5,6 \mathrm{mg} / \mathrm{dl}$ (Tabla 1 ).

Tabla 1. Análisis descriptivo de la muestra

\begin{tabular}{|c|c|c|c|}
\hline & $\begin{array}{c}\text { Total }(n=11) \\
\text { Media } \pm \text { DE }\end{array}$ & $\begin{array}{l}\text { Niños }(n=8) \\
\text { Media } \pm \text { DE }\end{array}$ & $\begin{array}{l}\text { Niñas }(n=3) \\
\text { Media } \pm \text { DE }\end{array}$ \\
\hline Edad (años) & $8,5 \pm 3,5$ & $9,4 \pm 3,9$ & $6,3 \pm 0,6$ \\
\hline HbA1c (\%) & $5,3 \pm 0,19$ & $5,3 \pm 0,2$ & $5,6 \pm 0$ \\
\hline Glucosa (mg/dl) & $86,6 \pm 5,6$ & $87,8 \pm 5,3$ & $82 \pm 5,7$ \\
\hline$\alpha$-hidroxibutirato $(\mu \mathrm{g} / \mathrm{ml})$ & $6,3 \pm 2,4$ & $6,4 \pm 2,7$ & $6,03 \pm 1,5$ \\
\hline Linoleil glicerofosfocolina $(\mu \mathrm{g} / \mathrm{ml})$ & $11,7 \pm 5,2$ & $10,2 \pm 2,7$ & $15,9 \pm 8,5$ \\
\hline Ácido oleico $(\mu \mathrm{g} / \mathrm{ml})$ & $53,9 \pm 29,0$ & $59 \pm 28,5$ & $40,2 \pm 31,2$ \\
\hline Insulina $(\mu \mathrm{U} / \mathrm{ml})$ & $13,6 \pm 8,6$ & $12,3 \pm 6,3$ & $17,2 \pm 14,2$ \\
\hline Puntuación Quantose IR ${ }^{\odot}$ & $78,09 \pm 9,25$ & $79,9 \pm 6,6$ & $73,3 \pm 15,2$ \\
\hline
\end{tabular}

aDE: Desviación estándar 
Por el contrario el test de metabolómica Quantose $I R{ }^{\odot}$, detectó 10 sujetos con resistencia a insulina tras el análisis del algoritmo de los 4 metabolitos y el resultado de su score (>63), Quantose $I R^{\odot}$ : 78,09 \pm 9,25 (Figura 1).

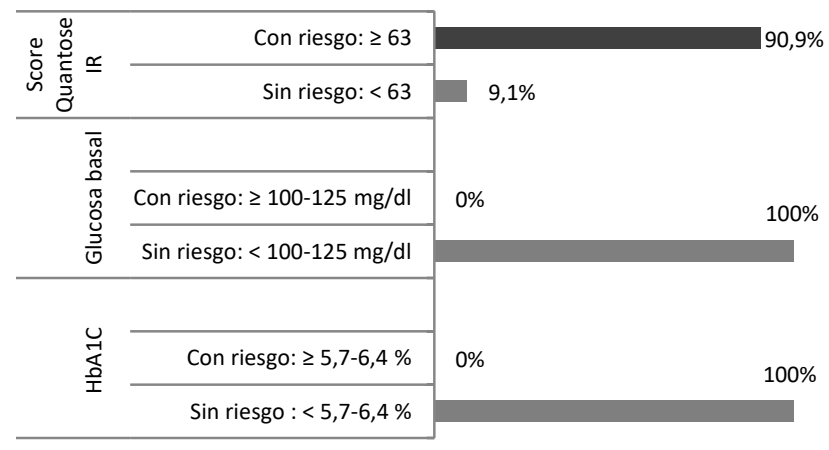

Figura 1. Resultados porcentuales obtenidos de los 3 biomarcadores principales. En cada uno de ellos, se muestra el porcentaje de sujetos estimados como riesgo o no riesgo según el test de Metabolomica Quantonse $I R^{\odot}$, glucosa basal, $\mathrm{HbA} 1 \mathrm{C}$

\section{Discusión}

Desafortunadamente, los métodos de detección generales para el descubrimiento de biomarcadores han tenido poco éxito. La metabolómica se está convirtiendo en una herramienta importante en el hallazgo de nuevos biomarcadores de diagnóstico y pronóstico(28). Scalbert, et al.(29), en su artículo sobre la identificación de biomarcadores en los que usó la metabolómica, incluye datos de apoyo sobre la validación de estos, sin embargo, la identificación de nuevos biomarcadores para detectar sujetos con riesgo de RI y para estratificar el riesgo de progresión a DM tipo 2 y/o enfermedad cardiovascular, con el fin de implementar estrategias efectivas para la prevención, así como para monitorizar la respuesta al tratamiento, sigue siendo una necesidad no satisfecha del todo(28).

Gall et al.(30) fueron los primeros en identificar como principal biomarcador de RI, el $\alpha$-hidroxibutirato; y como segundo biomarcador más importante la linoleil glicerofosfocolina. Debido a que estos marcadores biológicos no habían sido evaluados previamente en estudios clínicos, probaron su capacidad para predecir la DM tipo 2 en una cohorte observacional a largo plazo de sujetos en situación de riesgo, concluyendo que los niveles de $\alpha$ hidroxibutirato y linoleil glicerofosfocolina fueron predictivos de disglucemia.
Con base en los anteriores descubrimientos, se ha comprobado cómo Quantose ${ }^{T M}$ es una prueba sencilla e innovadora para la RI basada en una única muestra de sangre en ayunas y puede tener valor como un indicador temprano de riesgo para el desarrollo de prediabetes y DM tipo 2(22). Los tres metabolitos utilizados en la prueba, ácido $\alpha$-hidroxibutírico, ácido oleico y linoleil-glicerofosfocolina, tienen una serie de funciones potenciales en las vías metabólicas pertinentes a la acción de la insulina, la secreción de insulina y la función de las células $\beta$ del páncreas(22).

En el estudio de Cobb, et al.(22), los tres biomarcadores de la prueba mostraron un patrón similar de diferencias significativas para los sujetos resistentes a la insulina. Los niveles de ácido $\alpha$-hidroxibutírico y oleato aumentan en los sujetos resistentes a la insulina. Además, la puntuación Quantose ${ }^{\mathrm{TM}}$ es significativamente mejor para la detección de RI que la insulina sola (áreas bajo la cuerva de 0,79 vs. 0,74 ). A la hora de predecir la intolerancia a la glucosa, la puntuación Quantose ${ }^{\mathrm{TM}}$ también puede ser un indicador temprano. De hecho, la prueba identificó a los sujetos delgados en riesgo y con valores normales de glucosa en ayunas. La puntuaciones Quantose ${ }^{\mathrm{TM}} \mathrm{de}$ los progresores a la intolerancia a la glucosa disminuyeron en un 8\% en el transcurso de 3 años, indicando un incremento en la RI.

Ferrannini, et al.(31), reportaron niveles progresivamente más elevados de $\alpha$-hidroxibutírico y menores niveles de linoleil-glicerofosfocolina a través de los cuartiles de RI y en individuos con intolerancia a la glucosa o DM tipo 2. También demostraron dichos niveles al inicio del estudio en aquellos individuos con deterioro en comparación con una tolerancia estable a la glucosa después de 3 años de seguimiento y en aquellos individuos que progresan a DM tipo 2 después de 9,5 años de seguimiento. Cuando esto se añadió a un modelo para predecir la disglucemia o DM tipo 2, que incluía la historia familiar de diabetes, el sexo, la edad, el IMC, la glucosa en ayunas y los niveles en ayunas de estos dos metabolitos mejoraron la predictividad. Cuando el modelo incluía la glucosa en ayunas y a las 2 horas, los dos metabolitos tuvieron un impacto mínimo sobre la predictividad. Con estos resultados, Ferrannini et al.(31), confirmaron que los niveles de $\alpha$-hidroxibutírico y linoleilglicerofosfocolina en ayuno pueden ser nuevos biomarcadores para ayudar a predecir la disglucemia y la DM tipo 2. 


\section{Conclusiones}

Las pruebas basadas en la metabolómica podrían servir como un predictor temprano de la prediabetes o riesgo de DM tipo 2 en comparación con los marcadores tradicionales de glucemia (glucosa plasmática en ayunas, hemoglobina A1c, test de intolerancia oral a la glucosa), que aumentan relativamente tarde en la progresión de la enfermedad. A la luz de la actual pandemia mundial de diabetes tipo 2 , tales acciones preventivas son sumamente importantes.

Actualmente se están analizando más resultados clínicos de diversas poblaciones longitudinales para demostrar el potencial clínico completo de este nuevo test de RI y prediabetes.

La metabolómica tiene el potencial de proporcionar información sobre las rutas importantes para el metabolismo de la glucosa y la patogénesis de la DM tipo 2. También puede dilucidar biomarcadores capaces de mejorar la predicción del riesgo de deterioro de la tolerancia a la glucosa y diabetes tipo 2. La puntuación del test Quantose ${ }^{\mathrm{TM}}$ podría ser una herramienta útil en la práctica clínica para el seguimiento personalizado de la progresión hacia la prediabetes o DM tipo 2 del paciente y, mejorar así potencialmente su estado glucémico.

\section{Limitaciones del estudio}

Se trata de un estudio piloto, con una muestra muy reducida. Los autores son conscientes de que se debe seguir investigando con una población mayor e incorporando otros biomarcadores.

\section{Conflicto de intereses: Ninguno.}

\section{Referencias}

1. Manzur MdelR, Rodriguez S, Yañez RM, Ortuño M, García S, Fernandez N, et al. Síndrome metabólico, factores de riesgo en niños y adolescentes con sobrepeso. Gac méd Boliv [Internet]. 2016;39(2):94-8. Available from: http://www.scielo.org.bo/scielo.php?script=sci_arttext\&pid $=\mathrm{S} 1012-29662016000200008$

2. van der Aa MP, Fazeli Farsani S, Knibbe CAJ, de Boer A, van der Vorst MMJ. Population-Based Studies on the Epidemiology of Insulin Resistance in Children. J Diabetes Res. 2015;2015:1-9.

3. Fernando Carrasco N, José Eduardo Galgani F, Marcela Reyes J. Síndrome de resistencia a la insulina. Estudio y manejo. Rev Médica Clínica Las Condes. 2015;24(5):827-37.

4. Tokarz VL, MacDonald PE, Klip A. The cell biology of systemic insulin function. J Cell Biol. 2018;217(7):1-17.
5. Galgani JE, Ravussin E. Postprandial whole-body glycolysis is similar in insulin-resistant and insulin-sensitive non-diabetic humans. Diabetologia. 2012;55(3):737-42.

6. Jeffery SC, Hosking J, Jeffery AN, Murphy MJ, Voss LD, Wilkin TJ. Gender differences in insulin resistance during adolescence: A longitudinal study (EarlyBird) [Internet]. Vol. 59, Diabetologia. 2016. p. S547. Available from: http://ovidsp.ovid.com/ovidweb.cgi?T=JS\&PAGE=reference $\& D=$ emed18b\&NEWS $=\mathrm{N} \& A N=612314267$

7. Galera Martínez R, García García E, Vázquez López MÁ, Ortiz Pérez M, López Ruzafa E, Martín González M, et al. Factores asociados a insulinemia en población general adolescente. Nutr Hosp. 2013;28(5):1610-4.

8. Bajaj M, DeFronzo RA. Metabolic and molecular basis of insulin resistance. J Nucl Cardiol. 2003;10(3):311-23.

9. Weickert MO. What dietary modification best improves insulin sensitivity and why? Clin Endocrinol (Oxf). 2012;77(4):508-12.

10. Calero Bernal ML, Varela Aguilar JM. Diabetes tipo 2 infantojuvenil. Rev Clínica Española. 2018;218(7):372-81.

11. Manrique-Hurtado $\mathrm{H}$, Aro-Guardia $\mathrm{P}$, Pinto-Valdivia $\mathrm{M}$. Diabetes tipo 2 en niños. Serie de casos. Rev Medica Hered [Internet]. 2016;26(1):5-9. Available from: http://www.scielo.org.pe/pdf/rmh/v26n1/a02v26n1.pdf

12. Gómez-Ambrosi J, Rodríguez A, Catalán V, Frühbeck G. Papel del tejido adiposo en la inflamación asociada a la obesidad. Rev Esp Obes. 2008;6(5):264-79.

13. Kao KT, Sabin MA. Type 2 diabetes mellitus in children and adolescents. Aust Fam Physician. 2016;45(6):401-6.

14. Arisaka O, Sairenchi T, Ichikawa G, Koyama S. Increase of body mass index (BMI) from 1.5 to 3 years of age augments the degree of insulin resistance corresponding to BMI at 12 years of age. J Pediatr Endocrinol Metab. 2017;30(4):455-7.

15. Rosas-Sumano AB, Rodal-Canales FJ, Barrientos Pérez M, Cárdenas-Morales BE, Pérez-Campos Mayoral L, PérezCampos E. Hiperinsulinemia y resistencia insulínica en niños de dos escuelas públicas de Oaxaca, México. Rev Med Chil. 2016;144(8):1020-8.

16. International Diabetes Federation (IDF). IDF Diabetes Atlas Eighth edition 2017. In: Internatinal Diabetes Federation. 2017. p. 16-7.

17. Lehmann R. Diabetes subphenotypes and metabolomics: The key to discovering laboratory markers for personalized medicine? Clin Chem. 2013;59(9):1294-6.

18. Brown RJ, Yanovski JA. Estimation of insulin sensitivity in children: Methods, measures and controversies. Pediatr Diabetes. 2014;15(3):151-61.

19. Lentferink YE, Elst MAJ, Knibbe CAJ, van der Vorst MMJ. Predictors of Insulin Resistance in Children versus Adolescents with Obesity. J Obes [Internet]. 2017;2017:3793868. Available from: https://www.ncbi.nlm.nih.gov/pmc/articles/PMC5742469/

20. Katz A, Nambi SS, Mather K, Baron AD, Follmann DA, Sullivan G, et al. Quantitative insulin sensitivity check index: A simple, accurate method for assessing insulin sensitivity in humans. J Clin Endocrinol Metab. 2000;85(7):2402-10.

21. Gutch M, Kumar S, Razi S, Gupta K, Gupta A. Assessment of insulin sensitivity/resistance. Indian J Endocrinol Metab [Internet]. 2015;19(1):160. Available from: http://www.ijem.in/text.asp?2015/19/1/160/146874

22. Cobb J, Gall W, Adam KP, Nakhle P, Button E, Hathorn J, et al. A novel fasting blood test for insulin resistance and prediabetes. J Diabetes Sci Technol. 2013;7(1):100-10. 
23. Cobb J, Eckhart A, Motsinger-Reif A, Carr B, Groop L, Ferrannini E. $\alpha$-Hydroxybutyric acid is a selective metabolite biomarker of impaired glucose tolerance. Diabetes Care. 2016;39(6):988-95.

24. ADA. American Diabetes Association. 2. Classification and diagnosis of Diabetes: Standards of Medical Care in diabetes - 2019. Diabetes Care [Internet]. 2019;42(Suppl. 1):S13-28. Available from: https://doi.org/10.2337/dc19-S002

25. Gonzalez-Neira M, San Mauro-Martin I, Garcia-Angulo B, Fajardo D, Garicano-Vilar E. Nutritional and body composition assessment and its relationship with athletic performance in a women's soccer team. Rev Esp Nutr Humana Y Diet. 2015;19(1):36-48.

26. TANITA Corporation. Body Fat Moitor / Scale. Instruction Manual Tanita. Tanita Corporation of America Inc. 2011. p. 132.

27. Gunczler P. Síndrome de resistencia a la insulina en niños y adolescentes. Gac Med Caracas. 2006;114:62-70.
28. Milburn M V., Lawton KA. Application of Metabolomics to Diagnosis of Insulin Resistance. Annu Rev Med. 2013;64(1):291-305.

29. Scalbert A, Brennan L, Fiehn O, Hankemeier T, Kristal BS, van Ommen B, et al. Mass-spectrometry-based metabolomics: Limitations and recommendations for future progress with particular focus on nutrition research. Metabolomics. 2009;5(4):435-58.

30. Gall WE, Beebe K, Lawton KA, Adam KP, Mitchell MW, Nakhle PJ, et al. $\alpha$-hydroxybutyrate is an early biomarker of insulin resistance and glucose intolerance in a nondiabetic population. PLoS One. 2010;5(5):e10883.

31. Ferrannini E, Natali A, Camastra S, Nannipieri M, Mari A, Adam KP, et al. Early metabolic markers of the development of dysglycemia and type 2 diabetes and their physiological significance. Diabetes. 2013;62(5):1730-7. 